\title{
CONSERVATIVE MANAGEMENT OF CUTANEOUS FACIAL SINUS TRACT OF ODONTOGENIC ORIGIN: A CASE REPORT
}

\author{
Deepa. C1, Srinath K. S², Roopa K. S 3
}

HOW TO CITE THIS ARTICLE:

Deepa C, Srinath K. S, Roopa K. S. "Conservative management of cutaneous facial sinus tract of odontogenic origin: a case report". Journal of Evolution of Medical and Dental Sciences 2013; Vol2, Issue 31, August 5; Page: 5824-5829.

ABSTRACT: Odontogenic cutaneous sinuses are rare but well documented condition; however it is one of the most erroneously inferred lesions of face and neck region. The reason being its clinical presentation, as specific dental symptoms are absent in most of the cases, patients usually visit physicians first for the evaluation and treatment of such lesions. A delay by the attending clinician to correctly diagnose the primary dental etiology of the lesion has often resulted in multiple surgical excision and biopsies, long term antibiotic therapy, electrodessication and even radiation therapy, all of which have failed with recurrence of sinus tract. We report a case of cutaneous sinus tract related to a nonvital right lower premolar. The tooth had become nonvital because of trauma, resulting in pulpal necrosis and chronic periapical abscess that ultimately presented as cutaneous draining sinus tract along the right lower portion of the cheek. An appropriate treatment of this case resulted in successful resolution of the lesion, non-surgically.

KEYWORDS: Odontogenic, cutaneous, sinus tracts, periapical abscess, nonvital tooth.

INTRODUCTION: Extraoral-cutaneous sinus tracts of dental origin are chronically draining, fixed nodulo-cystic papule of the face and neck which continue to be of challenging diagnosis, as they do not always arise close to the underlying source of infection and dental symptoms are usually absent $^{1}$. The odontogenic sinus tract is a sequel to pathosis where the site of drainage can be intra or extraoral, depending on certain circumstances such as: the tooth involved, its apex position to muscular attachments, bacterial virulence, lower host resistance and the structures along the path of least resistance ${ }^{2}$. These cutaneous sinus tracts are most commonly located on the chin, the cheek or in the submandibular area and rarely in the nasal region, ${ }^{3}$ occurring more frequently from infected mandibular teeth than from infected maxillary teeth ${ }^{4}$ in a way making the diagnosis more difficult to the clinician. The radiographs play vital role in localizing the lesion, with the help of gutta percha (GP) points. A chronic periapical abscess is a long-standing, low grade infection of the periradicular alveolar bone, where the involved tooth is asymptomatic as the presence of the sinus tract precludes swelling or pain from pressure build up and it provides continued drainage of the periradicular lesion 4 . Based on literature reports, misdiagnosis has often worsened the chronicity of the lesion and has pronounced effects on facial aesthetics due to unnecessary treatments such as multiple biopsies, antibiotic regimens resulting in further skin scarring5. It has been observed that systemic antibiotic therapy will result in a temporary reduction of the drainage and apparent healing but definitive treatment is simple and effective, comprising of either tooth extraction or removal of infected pulp tissue with root canal therapy resulting in minimal scarring of the skin.

Here is a case of cutaneous sinus tract of dental origin which, following correct diagnosis healed thoroughly with endodontic therapy alone and did not require any surgical intervention. 
REPORT: A 24yr old female presented to the Department of Dentistry, R.L.J Hospital, Kolar with a complaint of cutaneous draining sinus on her right lower border of the cheek. History of alleged assault of a hard blow over her right jaw 5yrs back, intermittent swelling affecting the right lower face with discharge of pus since 6months but denied any history of pain. She suffered no other medical condition, was not on any medications and had no allergies.

Extraoral examination of the face and neck revealed no abnormalities, except for an area approximately $0.5 \mathrm{~cm}$ in diameter of sinus opening at the lower right portion of jaw. The opening of the lesion was crusted with minimal swelling. Palpation elicited purulent discharge and fixation of the lesion to underlying bone. Intraoral examination revealed no mucosal lesions or buccal sulcus swelling but elicited tenderness at root apex of 44. On hard tissue examination a mesio-buccally rotated 44 was seen that had no caries or periodontal involvement, but was tender to percussion. Investigations such as pulp vitality test and radiographs were taken; pulp sensitivity test gave a negative response. Extraoral PA view of mandible was taken instead of an intraoral periapical view because of short floor of mouth. Radiograph revealed an extensive periradicular radiolucency associated with apical region of 44, sinus tract link with periradicular lesion was confirmed by localization of the lesion radiographically using a GP point. A diagnosis of chronic periapical abcess with dento-cutaneous sinus tract was made. The patient was successfully treated with Root Canal Therapy where the necrotic pulp was extirpated using barbed broach later the canal was prepared with files and reamers using a step back technique irrigation with $3 \%$ sodium hypochlorite solution and saline. Then the root canal was filled with biocompatible material (gutta-percha root points and canal sealer) using the cold lateral condensation technique. Systemic antibiotics were advised during the procedure as an adjunct to aid in healing. There were no complications in the postoperative period. The cutaneous lesion showed complete healing but with a small depressed area over the region. The lesion healed within a month of treatment. A well healed periapical area was appreciated in a six months radiograph.

DISCUSSION: Cutaneous dental sinus or Odontogenic cutaneous sinus tract or Cutaneous facial sinus tract of dental origin was first stated by Winstock ${ }^{6}$ in 1950, later it was in 1980 the pathophysiology of spread of chronic dental infections was described by Kaban ${ }^{7}$, Sundqist ${ }^{8}$, demonstrated that pulpoperiapical diseases in traumatized teeth with intact crown and necrotic pulp can present with extraoral draining sinus as the only symptom, as featured in present case.

Draining sinuses are like channels which lead a dental focus of infection to be expressed onto the face/ neck. The point of drainage depends on location of apex of affected tooth in relation to muscular attachment to the bone and the path of least resistance through facial planes of face. As hypothesized by Seltzer S, the cutaneous sinus tract occurs when mandibular teeth apices of posterior teeth are anatomically inferior to the origin of buccinators muscle and if the apices are superior to buccinator origin, a buccal sulcus fistula/ more rarely, a lingual perforation might occur, more commonly seen in young individuals ${ }^{9}$. Kaban ${ }^{7} 1980$ elaborated path of spread of chronic dental infections occurred more commonly in mandible (80\%), than in maxilla (20\%) and the sinus located more commonly in the cheek and less commonly from canine space, nasolabial fold, nostrils and inner canthus of eyes. 
A characteristic cutaneous sinus appears as an erythematous, soft nodule fixed to the underlying structure with periodic drainage and crusting, lesion is depressed below normal skin surface which expresses pus on palpation of the surrounding tissue ${ }^{10}$.

Intraorally soft tissue seems normal and the source of infection could be a simple dental caries, periodontal disease, unerupted tooth, retained root fragment, tooth trauma, with or without fracture ${ }^{11}$. Vitality testing of potentially causal teeth gives negative response, other more confirmative test being the radiographic examination which presents as radiolucency of apex of infected tooth that can be traced from cutaneous orifice via the sinus tract to the source of lesion by gutta percha points, lachrymal probe or a diagnostic wire ${ }^{11}$.

Differential diagnosis of cutaneous draining sinus tracts includes osteomyelitis, congenital fistula, salivary gland fistula and infected cyst and deep mycotic infection. In addition, skin lesions such as pustules, furuncles, foreign-body lesions, squamous cell carcinoma and granulomatous disorders may all be similar superficially in appearance to a draining sinus tract of dental origin, but they are not true sinus tract ${ }^{12}$.

Once diagnosed root canal therapy or surgical extraction of the affected tooth is the treatment of choice ${ }^{13}$ and an antibiotic coverage acts as an adjunct to surgical therapy. Most authors believe that on removal of primary cause cutaneous lesion heals without any intervention within 5 to 14 days ${ }^{14}$, dimpling and hyperpigmentation of area occurs which fade over time and a surgical revision of bigger scar might be needed to provide better cosmetic result in future. Clinicians should be aware of the fact that cutaneous lesion of the face and neck can be of odontogenic origin and should seek communication and evaluation from appropriate specialist or general dental practitioners irrespective of whether or not associated with dental symptoms.

CONCLUSION: However advanced the medical line of treatment may get; one should not forget the fundamentals of a good decision making when it comes to diagnosing a disease. In the present case a detailed case history and clinical examination proved to be beneficial to the patient, which pinnacles the need for healthcare professionals to be aware of dentition as a probable cause of cutaneous sinuses of face and neck region. A traumatized tooth even in absence of caries or tooth fracture the clinician must investigate the pulpal health of the teeth in areas adjacent to cutaneous sinus, which helps in timely implementation of proper treatment and hence early resolution of lesion. The key to a successful treatment of such lesions must lay in healthy communication between the dentist and the physician in order to provide for timely recognition and treatment of rare cases.

\section{REFERENCES:}

1. Mittal N \& Gupta P. 2004. Management of Extra oral Sinus Cases: A clinical dilemma. J Endod, 30: 541-7.

2. Pasternak-Junior B, Teixeira CS, Silva-Sousa YTC \& Sousa-Neto MD. 2009. Diagnosis and treatment of odontogenic cutaneous sinus tracts of endodontic origin: three cases studies. Int Endod J, 42: 271-76. 
3. Heling I, Rotstein I. A persistent oronasal sinus tract of endodontic origin. J Endod 1989; 15:132-134.

4. McWalter GM, Alexander J B, delRio CE and Knott JW. Cutaneous sinus tracts of dental etiology. Oral Surgery Oral Medicine Oral Pathology 1988; 66:608-14.

5. Cantatore JL, Klein PA, Lieblich LM. Cutaneous dental sinus tract, a common misdiagnosis: a case report and review of the literature. Cutis; Cutaneous Medicine for the Practitioner 2002; 70(5): 264-265.

6. Winstock D. Four cases of external facial sinuses of dental origin. Proc R Soc Med 1959; 52:749-51.

7. Kaban LB. Draining skin lesions of dental origin: The path of spread of chronic odontogenic infection. Plast Reconster Surg 1980; 66:771-7.

8. Sundqvist G. 1976. Bacteriological studies of necrotic dental pulps (Dissertation). Umea University: Umea, Sweden.

9. Seltzer S. Endodontology: Biologic considerations in endodontic procedures. New York: McGraw Hill Book Co, 1971:167

10. Cutaneous sinuses of dental origin, Alibhai M, Balasundaram I, Bridle C. Cutaneous sinuses of dental origin. JSCR. 2012, 8:6.

11. Odontogenic sinus tracts. Ege Tip Dergisi 43 (2): 121 - 125, 2004.

12. Wood NK and Goaz PW. Differential diagnosis of oral lesions. $4^{\text {th }}$ edition. St. Louis: MosbyYear Book, 1991:264.

13. Hodges TP, Cohen DA, Deck D. Odontogenic sinus tracts. AM family practice 1989; 40: 113-6.

14. Spear KL, Sneddon PJ and Perry HO. Sinus tracts to the chin and jaw of dental origin. J Am Acad Dermatol 1983; 8:486-492.

\section{Pre-operative Photographs:}

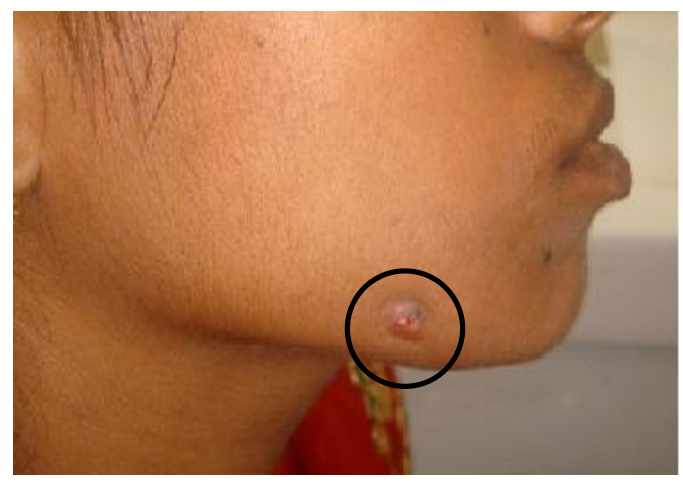

Extraoral sinus tract with pus discharge 


\section{CASE REPORT}

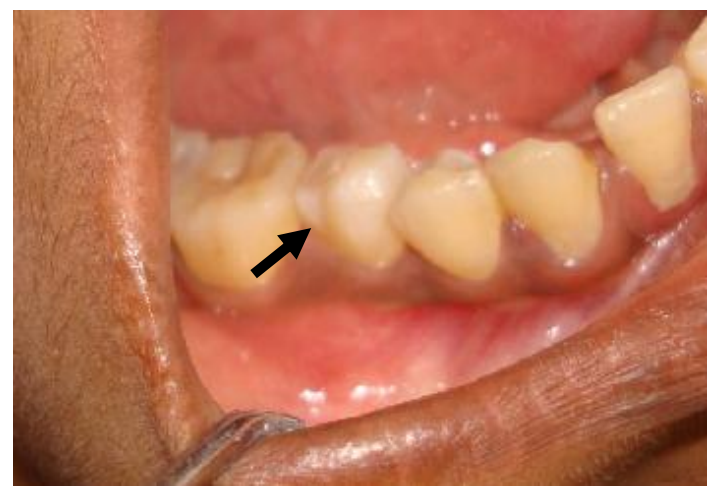

Intraoral sound crown structure of 45

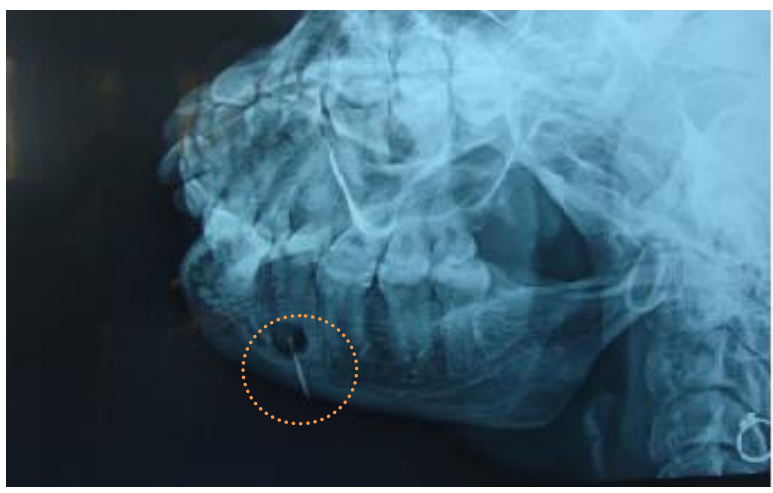

Radiograph showing preapical radiolucency being traced via gutta percha stick, near root apex of 45 .

\section{Six months Post-operative Photographs:}

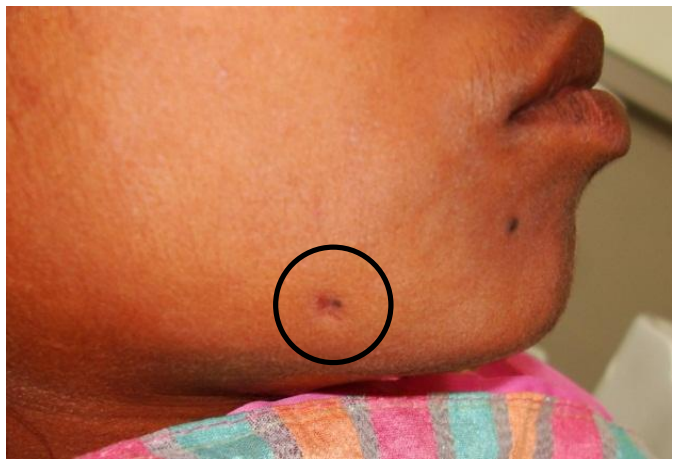

Fig 4: Completely healed extra oral sinus tract with minimal scarring. 


\section{CASE REPORT}

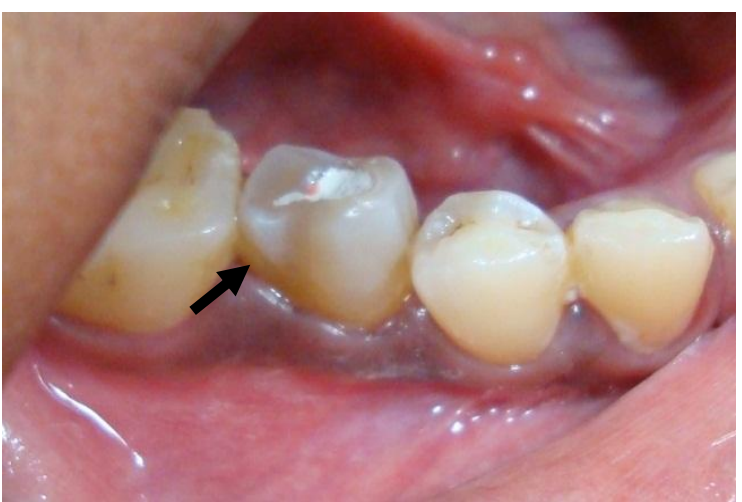

Root canal therapy of 45 showing restored crown.

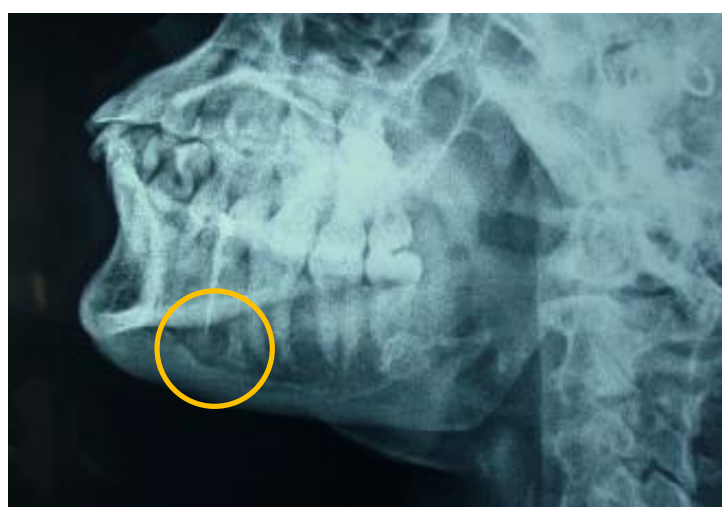

Radiograph showing radiopaque filling of 45 with reduced periapical rarefaction

\section{AUTHORS:}

1. Deepa C.

2. Srinath K.S.

3. Roopa K.S.

\section{PARTICULARS OF CONTRIBUTORS:}

1. Assistant Professor, Department of Dentistry, Sri Devraj Urs Medical College and Research Center.

2. Assistant Dental Surgeron, Department of Dentistry, Sri Devraj Urs Medical College and Research Center.

3. Junior Dentist, Department of Dentistry, Sri Devraj Urs Medical College and Research Center.

\section{NAME ADRRESS EMAIL ID OF THE} CORRESPONDING AUTHOR:

Dr. Deepa. C,

\# 461, $1^{\text {st }}$ Main, $8^{\text {th }}$ Cross,

Mico Colony, BTM $2^{\text {nd }}$ Stage,

Bangalore - 560076

Email -drdeepashekar@gmail.com

Date of Submission: 18/07/2013.

Date of Peer Review: 18/07/2013.

Date of Acceptance: 24/07/2013.

Date of Publishing: 31/07/2013 